PROCEEDINGS OF THE

AMERICAN MATHEMATICAL SOCIETY

Volume 27, No. 2, February 1971

\title{
LINEAR TRANSFORMATIONS UNDER WHICH THE DOUBLY STOCHASTIC MATRICES ARE INVARIANT ${ }^{1}$
}

\section{RICHARD SINKHORN}

Abstract. Let $\left[M_{n}(C)\right]$ denote the set of linear maps from the $n \times n$ complex matrices into themselves and let $\hat{\Omega}_{n}$ denote the set of complex doubly stochastic matrices, i.e. complex matrices whose row and column sums are 1 . If $F \in\left[M_{n}(C)\right]$ is such that $F\left(\hat{\Omega}_{n}\right) \subseteq \hat{\Omega}_{n}$ and $F^{*}\left(\hat{\Omega}_{n}\right) \subseteq \hat{\Omega}_{n}$, then there exist $A_{i}, B_{i}, A$, and $B \in \hat{\Omega}_{n}$ such that

$$
F(X)=\sum_{i} A_{i} X B_{i}+A X^{t} J_{n}+J_{n} X^{t} B-(1+m) J_{n} X J_{n}
$$

for all $n \times n$ complex matrices $X$, where $J_{n}$ is the $n \times n$ matrix whose elements are each $1 / n$ and where the superscript $t$ denotes transpose. $m$ denotes the number of the $A_{i}$ (or $B_{i}$ ).

Introduction. It has been of considerable interest to study linear maps from the $n \times n$ matrices to themselves that leave certain quantities invariant [1]-[12]. Often these maps are necessarily of the form $F(X)=A X B$ or $A X^{t} B$ with certain restrictions imposed on the $n \times n$ matrices $A$ and $B$, where the superscript $t$ denotes transpose. For example, Marcus and Moyls [8] show that such maps which preserve spectral values are of these forms with $A$ unimodular and $B=A^{-1}$. They show in [8], [9] that such maps which preserve certain given ranks are of these forms with $A$ and $B$ nonsingular. Marcus and May [7] show that such maps which preserve the permanent function are of these forms with $A=P_{1} D_{1}$ and $B=P_{2} D_{2}$ where the $P_{i}$ are permutation matrices and the $D_{i}$ are diagonal matrices such that per $D_{1} D_{2}=1$. Marcus, Minc, and Moyls [10] show that one may assume that $D_{1}$ $=D_{2}=I$ if in addition the linear map leaves the doubly stochastic matrices invariant.

This paper is concerned with linear transformations which map the set of $n \times n$ generalized doubly stochastic matrices, i.e. $n \times n$ complex matrices whose row and column sums are one, into itself. It is shown that the set of such maps $F$ which includes both $F$ and $F^{*}$ is precisely the set of linear combinations of transformations of the types $A X B$ and $C X^{t} D$, where the sum of the coefficients in any such

Received by the editors May 12, 1969.

AMS 1968 subject classifications. Primary 1565, 1585; Secondary 1530.

Key words and phrases. Faithful representation, generalized doubly stochastic matrices, Kroneker product, lexicographic ordering.

1 This research was partially supported by University of Houston grant FRSP (GIA) 69-27. 
combination is one and $A, B, C$, and $D$ are generalized doubly stochastic. It is clear that if $F$ is such a combination, $F\left(J_{n}\right)=F^{*}\left(J_{n}\right)$ $=J_{n}$, where $J_{n}$ is the $n \times n$ matrix whose entries are each $1 / n$. There are linear maps not of this form which send the generalized doubly stochastic matrices into themselves which do not have $J_{n}$ as a fixed point. For example, let $F_{1}$ be the linear map from the $2 \times 2$ complex matrices into themselves such that

$$
F_{1}\left(\begin{array}{ll}
a & b \\
c & d
\end{array}\right)=\left(\begin{array}{cc}
a+b & 0 \\
0 & c+d
\end{array}\right) .
$$

However, for such a map, the adjoint does not leave the generalized doubly stochastic matrices invariant.

We shall make use of the following notations and definitions. $M_{m n}(C)$ shall denote the $m \times n$ complex matrices, but we shall write $M_{n}(C)$ in case $m=n .0_{m n}$ is the zero matrix in $M_{m n}(C)$ whereas $0_{n}$ and $I_{n}$ are respectively the zero and identity matrix in $M_{n}(C) . E_{i j}$ $\in M_{m_{n}}(C)$ is a matrix whose element in the $(i, j)$ th position is 1 and whose elements are otherwise $0 . M_{n}(C)$ will be given the usual inner product: $(X, Y)=\sum_{i=1}^{n} \sum_{j=1}^{n} x_{i j} \bar{y}_{i j}$, where the bar denotes conjugation. The inner product induces the conventional norm on $M_{n}(C):\|x\|^{2}=(X, X) . \quad\left[M_{n}(C)\right]$ shall denote the set of linear maps of $M_{n}(C)$ into itself. The lexicographic representation of $X=\left(x_{i j}\right)$ $\in M_{n}(C)$ is the column vector

$$
x=\left(x_{11} x_{12} \cdots x_{1 n} x_{21} x_{22} \cdots x_{2 n} \cdots x_{n 1} x_{n 2} \cdots x_{n n}\right)^{t} .
$$

$F_{n^{2}}$ shall denote the $n^{2} \times n^{2}$ matrix representation of $F \in\left[M_{n}(C)\right]$ such that $F_{n^{2}} x=y$ whenever $F(X)=Y$, where $x$ and $y$ are the lexicographic representations of $X$ and $Y$, respectively. $F_{n^{2}}$ is called the faithful representation of $F$.

$\hat{\Omega}_{n}$ shall denote the $n \times n$ generalized doubly stochastic matrices. If $X_{k} \in M_{n_{k}}(C), k=1, \cdots, m$, and $n_{1}+\cdots+n_{m}=n$, the $n \times n$ matrix

$$
X_{1} \oplus X_{2} \oplus \cdots \oplus X_{m}=\left(\begin{array}{ccccc}
X_{1} & 0 & \cdots & 0 & 0 \\
0 & X_{2} & \cdots & 0 & 0 \\
\cdots & \cdots & \cdots & . & . \\
0 & 0 & \cdots & 0 & X_{m}
\end{array}\right)
$$

is called the direct sum of $X_{1}, \cdots, X_{m}$. The zeros indicate zero matrices of appropriate dimensions.

If $X \in M_{m n}(C)$ and $Y \in M_{p q}(C)$, the $m p \times n q$ matrix 


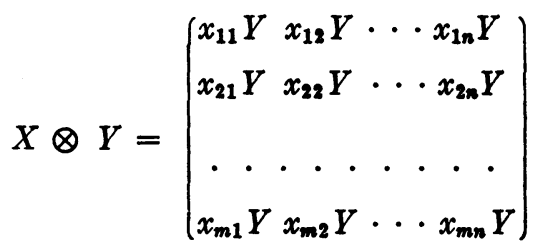

is called the Kroneker product of $X$ and $Y$.

The following well-known result is easily verified.

Theorem 1. Let $A, B \in M_{n}(C)$, and let $F \in\left[M_{n}(C)\right]$ be such that $F(X)=A X B$ for all $X \in M_{n}(C)$. Then $F_{n^{2}}=A \otimes B^{t}$ is the faithful representation of $F$.

\section{Preliminary results.}

Lemma 1. Let $A \in M_{(p r)(q s)}(C)$. There exist $A_{i} \in M_{p q}(C)$ and $B_{i}$ $\in M_{r s}(C)$ such that $A=\sum_{i}\left(A_{i} \otimes B_{i}\right)$.

Proof. Let the $A_{i}$ be the matrices $E_{i j} \in M_{p q}(C)$ listed in lexicographic order. Then write

$$
A=\left(\begin{array}{cccc}
A_{11} & A_{12} & \cdots & A_{1 q} \\
A_{21} & A_{22} & \cdots & A_{2 q} \\
\cdot & \cdot & \cdots & \cdot \\
A_{p 1} & A_{p 2} & \cdots & A_{p q}
\end{array}\right),
$$

where each $A_{i j} \in M_{r s}(C)$, and let the $B_{i}$ be the $A_{i j}$ arranged in lexicographic order. Clearly $A=\sum_{i}\left(A_{i} \otimes B_{i}\right)$.

TheOREM 2. Suppose that $Q \in M_{p q}(C), R \in M_{r s}(C)$, and $S$ $\in M_{(p r)(q s)}(C)$. There exist $M_{i} \in M_{p q}(C)$ and $N_{i} \in M_{r s}(C)$ such that $Q=\sum_{i} M_{i}, R=\sum_{i} N_{i}$, and $S=\sum_{i}\left(M_{i} \otimes N_{i}\right)$.

Proof. By Lemma 1 there are $A_{i} \in M_{p q}(C)$ and $B_{i} \in M_{r q}(C)$ such that $S-(Q \otimes R)=\sum_{i}\left(A_{i} \otimes B_{i}\right)$. Then

and

$$
\begin{aligned}
& Q=Q+\sum_{i} A_{i}+\sum_{i} 0_{p q}+\sum_{i}\left(-A_{i}\right), \\
& R=R+\sum_{i} B_{i}+\sum_{i}\left(-B_{i}\right)+\sum_{i} 0_{r z}
\end{aligned}
$$

$$
\begin{aligned}
S= & (Q \otimes R)+\sum_{i}\left(A_{i} \otimes B_{i}\right)+\sum_{i}\left(0_{p q} \otimes\left(-B_{i}\right)\right) \\
& +\sum_{i}\left(\left(-A_{i}\right) \otimes 0_{r s}\right) .
\end{aligned}
$$


Lemma 2. Let $A, B \in M_{n}(C)$, and let $F \in\left[M_{n}(C)\right]$ be such that $F(X)=A X B$ for all $X \in M_{n}(C)$. Then $F^{*}(X)=A^{*} X B^{*}$ for all $X$ $\in M_{n}(C)$, where $F^{*}$ is the adjoint of $F$ and $A^{*}$ and $B^{*}$ are respectively the conjugate transposes of $A$ and $B$.

PROOF. This follows from the fact that $F_{n^{2}}^{*}=\left(A \otimes B^{t}\right)^{*}=A^{*} \otimes B^{*^{t}}$.

LEMMA 3. Let $U$ be a real unitary matrix in $M_{n}(C)$ with first column $(1 / \sqrt{ } n)(1,1, \cdots, 1)^{t}$. Define $H \in\left[M_{n}(C)\right]$ by $H(X)=U^{t} X U$ for all $X \in M_{n}(C)$. Then $H$ is unitary, and for each $M \in \hat{\Omega}_{n}$, there is an $M^{\prime}$ $\in M_{n-1}(C)$ such that $H(M)=1 \oplus M^{\prime}$.

Proof. $H$ is unitary by Lemma 2.

For $M \in \hat{\Omega}_{n}$ put $W=H(M)$. Then

$$
w_{1 j}=\sum_{i=1}^{n} \sum_{k=1}^{n} u_{i 1} m_{i k} u_{k j}=\frac{1}{\sqrt{ } n} \sum_{k=1}^{n} \sum_{i=1}^{n} m_{i k} u_{k j}=\frac{1}{\sqrt{ } n} \sum_{k=1}^{n} u_{k j}=\delta_{1 j},
$$

Kroneker's delta. Similarly, $w_{i 1}=\delta_{i \mathbf{1}}$.

LEMMA 4. Let $P \in M_{n^{2}}(C)$ be the permutation matrix such that for any $A, B \in M_{n-1}(C)$,

$$
P[(1 \oplus A) \otimes(1 \oplus B)] P^{t}=1 \oplus A \oplus B \oplus(A \otimes B),
$$

and let $T$ denote the transpose map. Then

$$
P T_{n}: P^{t}=1 \oplus\left(\begin{array}{ll}
0_{n-1} & I_{n-1} \\
I_{n-1} & 0_{n-1}
\end{array}\right) \oplus T_{(n-1)^{2}}
$$

Proof. Put

$$
V=1 \oplus\left(\begin{array}{ll}
0_{n-1} & I_{n-1} \\
I_{n-1} & 0_{n-1}
\end{array}\right) \oplus T_{(n-1)^{2}}
$$

Let $\sigma, \tau$, and $\omega$ respectively denote permutations of $1, \cdots, n^{2}$ such that $p_{i \sigma(i)}=t_{i \tau(i)}=v_{i \omega(i)}=1$ for $i=1, \cdots, n^{2}$, where $P=\left(p_{i j}\right), T_{n^{2}}$ $=\left(t_{i j}\right)$, and $V=\left(v_{i j}\right)$. Then

$$
\begin{aligned}
\sigma(k)= & (k-1) n+1, \quad k=1, \cdots, n: \\
\sigma[k(n-1)+j]= & (k-1) n+j, \\
& j=2, \cdots, n, \quad k=1, \cdots, n, \\
\tau[(k-1) n+j]= & (j-1) n+k, \\
& j=1, \cdots, n, \quad k=1, \cdots, n,
\end{aligned}
$$

and 
(3)

$$
\begin{array}{rlrl}
\omega(1) & =1 ; \quad \omega(k)=n+k-1, & k & =2, \cdots, n \\
\omega(n+j-1) & =j, & & j=2, \cdots, n \\
\omega[k(n-1)+j+n] & =j(n-1)+k+n, & \\
j & =1, \cdots, n-1, \quad k=1, \cdots, n-1 .
\end{array}
$$

If $\quad k=1, \cdots, n, \quad \tau \sigma(k)=\tau[(k-1) n+1]=(1-1) n+k=k ; \quad$ if $j=2, \cdots, n, \quad k=1, \cdots, n, \quad \tau \sigma[k(n-1)+j]=\tau[(k-1) n+j]=$ $(j-1) n+k$. Also $\sigma \omega(1)=\sigma(1)=1$, while if $k=2, \cdots, n, \sigma \omega(k)$ $=\sigma(n+k-1)=(1-1) n+k=k$; if $j=2, \cdots, n, \sigma \omega(n-1+j)=\sigma(j)$ $=(j-1) n+1$; if $j=2, \cdots, n, k=2, \cdots, n$,

$$
\begin{aligned}
\sigma \omega[k(n-1)+j] & =\sigma \omega[(k-1)(n-1)+(j-1)+n] \\
& =\sigma[(j-1)(n-1)+(k-1)+n] \\
& =\sigma[j(n-1)+k]=(j-1) n+k .
\end{aligned}
$$

Thus $\tau \sigma(k)=\sigma \omega(k)$ for $k=1, \cdots, n^{2}$, and therefore $P T_{n^{2}}=V P$.

LEMma 5. If $W \in \hat{\Omega}_{n}$, then $\left\|W-J_{n}\right\|^{2}+1=\|W\|^{2}$.

Proof. $\quad\left\|W-J_{n}\right\|^{2}=\left(W-J_{n}, W-J_{n}\right)=(W, W)-\left(W, J_{n}\right)-$ $\left(J_{n}, W_{n}\right)+\left(J_{n}, J_{n}\right)=\|W\|^{2}-1-1+1=\|W\|^{2}-1$.

It follows that for all $W \in \hat{\Omega}_{n},\|W\| \geqq 1$, and equality holds if and only if $W=J_{n}$.

Corollary. If $F \in\left[M_{n}(C)\right]$ is such that $F\left(\hat{\Omega}_{n}\right) \subseteq \hat{\Omega}_{n}$ and $F^{*}\left(\hat{\Omega}_{n}\right)$ $\subseteq \widehat{\Omega}_{n}$, then necessarily $F\left(J_{n}\right)=F^{*}\left(J_{n}\right)=J_{n}$.

Proof. Suppose that $F\left(J_{n}\right)=W$ and $F^{*}\left(J_{n}\right)=X$. Put $F(X)=Y$ and $F^{*}(W)=Z$. Then $W, X, Y$, and $Z \in \hat{\Omega}_{n}$, and

$$
\|W\|^{2}=(W, W)=\left(F\left(J_{n}\right), W\right)=\left(J_{n}, F^{*}(W)\right)=\left(J_{n}, Z\right)=1 ;
$$

whence $W=J_{n}$. Likewise $\|X\|^{2}=\left(X, F^{*}\left(J_{n}\right)\right)=\left(Y, J_{n}\right)=1$, and so $X=J_{n}$.

Consequences. Let $K, L \in\left[M_{n}(C)\right]$ be defined respectively by $K(X)=A X B$ and $L(X)=A X^{t} B$, where $A$ and $B \in \hat{\Omega}_{n}$ are fixed. Let $U$ and $H$ be as in Lemma 3 . There exist matrices $A^{\prime}, B^{\prime} \in M_{n-1}(C)$ such that $U^{t} A U=1 \oplus A^{\prime}$ and $U^{t} B U=1 \oplus B^{\prime}$. Then, since $H^{*}(X)=U X U^{t}$ for any $X \in M_{n}(C)$,

$$
\left(H K H^{*}\right)(X)=\left(U^{t} A U\right) X\left(U^{t} B U\right)=\left(1 \oplus A^{\prime}\right) X\left(1 \oplus B^{\prime}\right) .
$$

Thus $\left(H K H^{*}\right)_{n^{2}}=\left(1 \oplus A^{\prime}\right) \otimes\left(1 \oplus B^{\prime t}\right)$, and so

$$
P\left(H K H^{*}\right)_{n}^{2} P^{t}=1 \oplus A^{\prime} \oplus B^{\prime t} \oplus\left(A^{\prime} \otimes B^{\prime}\right),
$$


where $P$ is as in Lemma 4.

Also if $T$ is the transpose map of Lemma 4, $\left(K L H^{*}\right)(X)=\left(B K T H^{*}\right)(X)=\left(U^{t} A U\right) X^{t}\left(U^{t} B U\right)=\left(H K H^{*} T\right)(X)$.

Whence $\left(H L H^{*}\right)_{n^{2}}=\left(H K H^{*}\right)_{n^{2}} T_{n^{2}}$, and so, by Lemma 4,

$$
\begin{aligned}
P\left(B L H^{*}\right)_{n}{ }^{2} P^{t}= & P\left(H K H^{*}\right)_{n}{ }^{2} P^{t} P T_{n^{2}} P^{t} \\
= & \left(1 \oplus A^{\prime} \oplus B^{\prime t} \oplus\left(A^{\prime} \otimes B^{\prime t}\right)\right) \\
& \cdot\left(1 \oplus\left(\begin{array}{ll}
0_{n-1} & I_{n-1} \\
I_{n-1} & 0_{n-1}
\end{array}\right) \oplus T_{(n-1)^{2}}\right) \\
= & 1 \oplus\left(\begin{array}{ll}
0_{n-1} & A^{\prime} \\
B^{\prime t} & 0_{n-1}
\end{array}\right) \oplus\left(A^{\prime} \otimes B^{\prime t}\right) T_{(n-1)^{2}} .
\end{aligned}
$$

Note that the component $A^{\prime} \otimes B^{\prime t}$ represents the reduced map $K^{\prime}(Y)=A^{\prime} Y B^{\prime}$ and $\left(A^{\prime} \otimes B^{\prime t}\right) T_{(n-1)^{2}}$ represents the reduced map $L^{\prime}(Y)=A^{\prime} Y^{\prime} B^{\prime}$, where $K^{\prime}, L^{\prime} \in\left[M_{n-1}(C)\right]$.

Suppose that $F \in\left[M_{n}(C)\right]$ is such that $F\left(\hat{\Omega}_{n}\right) \subseteq \hat{\Omega}_{n}$ and $F^{*}\left(\hat{\Omega}_{n}\right) \subseteq \hat{\Omega}_{n}$. By the corollary to Lemma $5, F\left(J_{n}\right)=F^{*}\left(J_{n}\right)=J_{n}$. Since $H\left(J_{n}\right)$ $=U^{t} J_{n} U=1 \oplus 0_{n-1},\left(H F H^{*}\right)\left(1 \oplus 0_{n-1}\right)=1 \oplus 0_{n-1}$.

Let $W, X \in \hat{\Omega}_{n}$ and put $Y=F(W), Z=F^{*}(X)$. There are matrices $W^{\prime}, X^{\prime}, Y^{\prime}$, and $Z^{\prime} \in M_{n-1}(C)$ such that $H(W)=1 \oplus W^{\prime}, H(X)$ $=1 \oplus X^{\prime}, H(Y)=1 \oplus Y^{\prime}$, and $H(Z)=1 \oplus Z^{\prime}$. It follows that

$$
\left(H F H^{*}\right)\left(1 \oplus W^{\prime}\right)=H F(W)=H(Y)=1 \oplus Y^{\prime}
$$

and thus that

$$
\begin{aligned}
\left(H F H^{*}\right)\left(0 \oplus W^{\prime}\right) & =\left(H F H^{*}\right)\left\{\left(1 \oplus W^{\prime}\right)-\left(1 \oplus 0_{n-1}\right)\right\} \\
& =\left(1 \oplus Y^{\prime}\right)-\left(1 \oplus 0_{n-1}\right)=0 \oplus Y^{\prime} .
\end{aligned}
$$

Likewise, $\left(H F H^{*}\right)^{*}\left(1 \oplus X^{\prime}\right)=1 \oplus Z^{\prime}$ and $\left(H F H^{*}\right)^{*}\left(0 \oplus X^{\prime}\right)=0 \oplus Z^{\prime}$. If $w^{\prime \prime}, x^{\prime \prime}, y^{\prime \prime}$ and $z^{\prime \prime}$ are the lexicographic representations of $1 \oplus W^{\prime}$, $1 \oplus X^{\prime}, 1 \oplus Y^{\prime}$, and $1 \oplus Z^{\prime}$, and similarly for $w^{\prime \prime \prime}, x^{\prime \prime \prime}, y^{\prime \prime \prime}, z^{\prime \prime \prime}$ and $0 \oplus W^{\prime}, 0 \oplus X^{\prime}, 0 \oplus Y^{\prime}, 0 \oplus Z^{\prime}$, then

$$
\begin{aligned}
& \left(H F H^{*}\right)_{n^{2} w^{\prime \prime}}=y^{\prime \prime}, \quad\left(H F H^{*}\right)^{*}{ }^{2} x^{\prime \prime}=z^{\prime \prime}, \\
& \left(B F H^{*}\right)_{n}{ }^{22 w^{\prime \prime \prime}}=y^{\prime \prime \prime}, \quad\left(B F H^{*}\right)^{*}{ }_{n} x^{\prime \prime \prime}=z^{\prime \prime \prime},
\end{aligned}
$$

where $\left(H F H^{*}\right)^{*}{ }_{n^{2}}$ is the conjugate transpose of $\left(H F H^{*}\right)_{n^{2} \text {. }}$

Note that $P w^{\prime \prime}=\left(1, \theta^{t}, w^{t}\right)^{t}, P w^{\prime \prime \prime}=\left(0, \theta^{t}, w^{t}\right)^{\imath}$, and similarly for $x^{\prime \prime}, x^{\prime \prime \prime}, y^{\prime \prime}, y^{\prime \prime \prime}$, and $z^{\prime \prime}, z^{\prime \prime \prime}$, where $\theta$ is a $2(n-1)$ dimensional column of zeros and where $w^{\prime}, x^{\prime}, y^{\prime}$, and $z^{\prime}$ are respectively the lexicographic representations of $W^{\prime}, X^{\prime}, Y^{\prime}$, and $Z^{\prime}$.

Put 


$$
P\left(H F H^{*}\right)_{n}{ }^{2} P^{t}=\left(\begin{array}{lll}
F_{0} & F_{12} & F_{13} \\
F_{21} & F_{1} & F_{23} \\
F_{31} & F_{32} & F_{2}
\end{array}\right),
$$

where $F_{0}$ is $1 \times 1, F_{1}$ is $2(n-1) \times 2(n-1)$, and $F_{2}$ is $(n-1)^{2} \times(n-1)^{2}$. Then

$$
\begin{aligned}
& \left(\begin{array}{lll}
F_{0} & F_{12} & F_{13} \\
F_{21} & F_{1} & F_{23} \\
F_{31} & F_{32} & F_{2}
\end{array}\right)\left(\begin{array}{c}
1 \\
\theta \\
w^{\prime}
\end{array}\right)=\left(\begin{array}{c}
1 \\
\theta \\
y^{\prime}
\end{array}\right), \quad\left(\begin{array}{lll}
F_{0} & F_{12} & F_{13} \\
F_{21} & F_{1} & F_{23} \\
F_{31} & F_{32} & F_{2}
\end{array}\right)^{*}\left(\begin{array}{c}
1 \\
\theta \\
x^{\prime}
\end{array}\right)=\left(\begin{array}{c}
1 \\
\theta \\
z^{\prime}
\end{array}\right), \\
& \left(\begin{array}{lll}
F_{0} & F_{12} & F_{13} \\
F_{21} & F_{1} & F_{23} \\
F_{31} & F_{32} & F_{2}
\end{array}\right)\left(\begin{array}{c}
0 \\
\theta \\
w^{\prime}
\end{array}\right)=\left(\begin{array}{c}
0 \\
\theta \\
y^{\prime}
\end{array}\right), \quad\left(\begin{array}{lll}
F_{0} & F_{12} & F_{13} \\
F_{21} & F_{1} & F_{28} \\
F_{31} & F_{32} & F_{2}
\end{array}\right)^{*}\left(\begin{array}{l}
0 \\
\theta \\
x^{\prime}
\end{array}\right)=\left(\begin{array}{c}
0 \\
\theta \\
z^{\prime}
\end{array}\right) .
\end{aligned}
$$

The third equation in (1) indicates that $F_{13}$ and $F_{23}$ are zero; the fourth equation indicates that $F_{31}$ and $F_{82}$ are zero. Given these facts, the first equation indicates that $F_{0}=1$ and $F_{21}=\theta$. The second equation indicates that $F_{0}=1$ and $F_{12}=\theta^{t}$. Whence

$$
P\left(H F H^{*}\right)_{n}^{2} P^{t}=1 \oplus F_{1} \oplus F_{2} \text {. }
$$

If we write

$$
F_{1}=\left(\begin{array}{ll}
Q & Q^{\prime} \\
R^{\prime t} & R
\end{array}\right)
$$

where $Q, Q^{\prime}, R$, and $R^{\prime} \in M_{n-1}(C)$, we have

(2) $P\left(B F H^{*}\right)_{n} P^{t}=\left(1 \oplus Q \oplus R \oplus F_{2}\right)+\left(0 \oplus\left(\begin{array}{cc}0_{n-1} & Q^{\prime} \\ R^{\prime t} & 0_{n-1}\end{array}\right) \oplus 0_{(n-1)^{2}}\right)$.

Let $L_{1}, L_{2} \in\left[M_{n}(C)\right]$ be respectively defined by $L_{1}(X)=A X^{t} J_{n}$ and $L_{2}(X)=J_{n} X^{t} B$ where $A=H^{*}\left(1 \oplus Q^{\prime}\right) \in \hat{\Omega}_{n}$ and $B=H^{*}\left(1 \oplus R^{\prime}\right) \in \hat{\Omega}_{n}$. Then if $J(X)=J_{n} X J_{n}$ for all $X \in M_{n}(C)$,

$$
\begin{aligned}
\left(0 \oplus\left(\begin{array}{cc}
0_{n-1} & Q^{\prime} \\
R^{\prime t} & 0_{n-1}
\end{array}\right) \oplus 0_{(n-1)^{2}}\right) & =\left(1 \oplus\left(\begin{array}{cc}
0_{n-1} & Q^{\prime} \\
0_{n-1}^{t} & 0_{n-1}
\end{array}\right) \oplus\left(Q^{\prime} \otimes 0_{n-1}^{t}\right) T_{(n-1)^{2}}\right) \\
& +\left(1 \oplus\left(\begin{array}{cc}
0_{n-1} & 0_{n-1} \\
R^{\prime t} & 0_{n-1}
\end{array}\right) \oplus\left(0_{n-1} \otimes R^{\prime t}\right) T_{(n-1)^{2}}\right) \\
& -2\left(1 \oplus\left(\begin{array}{cc}
0_{n-1} & 0_{n-1} \\
0_{n-1} & 0_{n-1}^{t}
\end{array}\right) \oplus\left(0_{n-1} \otimes 0_{n-1}^{t}\right)\right) \\
= & P\left(H L_{1} H^{*}\right)_{n^{2}} P^{t}+P\left(H L_{2} H^{*}\right)_{n}^{2} P^{t}-2 P\left(H J H^{*}\right)_{n}^{2} P^{t} .
\end{aligned}
$$


By Theorem 2, there exist matrices $M_{i}$ and $N_{i} \in M_{n-1}(C)$ such that $Q=\sum_{i} M_{i}, R=\sum_{i} N_{i}^{t}$, and $F_{2}=\sum_{i}\left(M_{i} \otimes N_{i}^{t}\right)$. For each $i$ let $A_{i}$ $=H^{*}\left(1 \oplus M_{i}\right)$ and $B_{i}=H^{*}\left(1 \oplus N_{i}\right)$. Then each $A_{i}$ and $B_{i} \in \hat{\Omega}_{n}$. Let $K_{i} \in\left[M_{n}(C)\right]$ be defined by $K_{i}(X)=A_{i} X B_{i}$ for all $X \in M_{n}(C)$.

Then

$1 \oplus Q \oplus R \oplus F_{2}=\left(1 \oplus \sum_{i} M_{i} \oplus \sum_{i} N_{i}^{t} \oplus \sum_{i}\left(M_{i} \otimes N_{i}^{t}\right)\right)$

$$
\begin{aligned}
= & \sum_{i}\left(1 \oplus M_{i} \oplus N_{i}^{t} \oplus\left(M_{i} \otimes N_{i}^{t}\right)\right) \\
& +(1-m)\left(1 \oplus 0_{n^{2}-1}\right) \\
= & \sum_{i} P\left(H K_{i} H^{*}\right)_{n}{ }^{2} P^{t}+(1-m) P\left(H J H^{*}\right)_{n}{ }^{2} P^{t}
\end{aligned}
$$

where $m$ is the number of $M_{i}$ (or $N_{i}$ ).

It follows from (2), (3), and (4) that

$$
F=\sum_{i} K_{i}+L_{1}+L_{2}-(1+m) J .
$$

In summary,

THEOREM 3. Let $F \in\left[M_{n}(C)\right]$ be such that $F\left(\hat{\Omega}_{n}\right) \subseteq \hat{\Omega}_{n}$ and $F^{*}\left(\hat{\Omega}_{n}\right)$ $\subseteq \hat{\Omega}_{n}$. Then there exist matrices $A_{i}, B_{i}, A$, and $B \in \hat{\Omega}_{n}$ such that

$$
F(X)=\sum_{i} A_{i} X B_{i}+A X^{t} J_{n}+J_{n} X^{i} B-(1+m) J_{n} X J_{n}
$$

for all $X \in M_{n}(C)$, where $m$ is the number of $A_{i}\left(\right.$ or $\left.B_{i}\right)$.

The author is most grateful for an invaluable discussion with Professor Paul Knopp concerning this presentation.

\section{REFERENCES}

1. E. P. Botta, Linear transformations on matrices: The invariance of a class of general matrix functions, Canad. J. Math. 19 (1967), 281-290. MR 35 \#1609.

2. - Linear transformations on matrices: The invariance of a class of general matrix functions. II, Canad. J. Math. 20 (1968), 739-748.

3. J. Dieudonné, Sur une généralisation du groupe orthogonal à quatre variables, Arch. Math. 1 (1949), 282-287. MR 10, 586.

4. L. K. Hua, Geometries of matrices. I. Generalizations of von Staudt's theorem, Trans. Amer. Math. Soc. 57 (1945), 441-481. MR 7, 58.

5. H. G. Jacob, Jr., Coherence invariant mappings on Kronecker products, Amer. J. Math. 77 (1955), 177-189. MR 16, 667.

6. M. Marcus, Linear operations on matrices, Amer. Math. Monthly 69 (1962), 837-847. MR 26 \#5007.

7. M. Marcus and F. C. May, The permanent function, Canad. J. Math. 14 (1962), 177-189. MR 25 \#1178. 
8. M. Marcus and B. N. Moyls, Linear transformations on algebras of matrices, Canad. J. Math. 11 (1959), 61-66. MR 20 \#6432.

9. - Transformations on tensor product spaces, Pacific J. Math. 9 (1959), 1215-1221. MR 21 \#7219.

10. M. Marcus, H. Minc and B. N. Moyls, Permanent preservers on doubly stochastic matrices, Canad. J. Math. 14 (1962), 190-194.

11. M. Marcus and R. Purves, Linear transformations on algebras of matrices: The invariance of the elementary symmetric functions, Canad. J. Math. 11 (1959), 383396. MR 21 \#4167.

12. K. Morita, Schwarz's lemma in a homogeneous space of higher dimensions, Japan J. Math. 19 (1944), 45-56. MR 7, 381.

University of Houston, Houston, Texas 77004 